\title{
Effects of Moderate Exercise on the
} Biochemical, Physiological, Morphological and Functional Parameters of the Aorta in the Presence of Estrogen Deprivation and Dyslipidemia: an Experimental Model

\author{
Claudia Marchon ${ }^{a}$ Elisabete de Marco Ornelas ${ }^{a}$ Katia Aparecida da Silva Viegas ${ }^{b}$ \\ Silvia Lacchinib Romeu Rodrigues de Souza ${ }^{\mathrm{b}}$ Fernando Luiz Affonso Fonseca,d \\ Laura Beatriz Mesiano Maifrino a,e
}

aLaboratório de Estudos Morfoquantitativo e Imunohistoquímico da Universidade São Judas Tadeu, São Paulo, ' Instituto de Ciências Biomédicas da Universidade de São Paulo, São Paulo, 'Universidade Federal de São Paulo, Diadema, SP, 'Faculdade de Medicina do Abc, Santo André, SP, eInstituto Dante Pazzanese de Cardiologia, São Paulo, Brasil

\section{Key Words}

Moderate exercise $\cdot$ Aorta $\bullet$ Estrogen $\cdot$ Deprivation $・$ Dislipydemia

\begin{abstract}
Background: The estrogen deficiency, abnormal lipid profile, weight gain and a sedentary lifestyle are factors associated with the increased prevalence of cardiovascular disease in menopausal women. However, physical exercise practice reduces some of these risk factors. Moreover, it has been shown that exercise has an impact on inflammation, in sympathetic activity and improves endothelial function. Aims: The present study aims to evaluate the effects of moderate aerobic training on biochemical, morphological and physiological parameters in LDL Knockout mice with estrogen deprivation, evaluating the components of the ascending aortic wall. Methods: The animals were randomly divided into six groups $(n=5)$ : sedentary control (SC), sedentary control ovariectomized (SCO), trained control ovariectomized (TCO), LDL-Knockout sedentary (KS), LDL-Knockout sedentary ovariectomized (KOS) and LDLKnockout trained ovariectomized (KOT). The trained groups underwent a protocol of moderate training for 4 weeks on a treadmill with speed and progressive load. After training, blood samples were collected for biochemical assessments and the aorta was removed for dissection and histological morphometry study. In addition, the expression of angiotensin-converting enzyme (ACE) and angiotensin II proteins were examined by immunohistochemistry in all groups of animals. Results: Changes of expressions of ACE and angiotensin II were found when the group was subjected to exercise. The concentrations of cholesterol and triglycerides were lower in the groups of animals with estrogen deprivation and dyslipidemia. In animals
\end{abstract}

Fernando Luiz Affonso Fonseca

KARGER 125
Laboratório de Análises Clínicas da FMABC, Faculdade de Medicina do ABC Av. Principe de Gales, n. 821, Santo André, SP, CEP: 09060-650, (Brasil)

Tel. +551149935488 Cel: +5511981587419, E-Mail profferfonseca@gmail.com 
that performed exercises we found significant increase $(p<0.05)$ in $\mathrm{Vv}[\mathrm{lam}]$; decrease in $\mathrm{Vv}$ [col] and CWT, and a tendency for decrease both in TS and IMT when compared to the SC groups. The histological morphometry findings showed consistency in the results of the aorta study when the ovariectomized group underwent the exercise protocol. Conclusion: We conclude that physical training contributed to reducing vessel rigidity and to improvements in vascular compliance, with the increase in volume density of elastic lamellae in the estrogen-deprived groups who had normal cholesterol levels.

Copyright $@ 2015$ S. Karger AG, Basel

\section{Introduction}

It is believed that estrogen deficiency, abnormal lipid profile, weight gain and a sedentary lifestyle are the main factors associated with a higher prevalence of cardiovascular disease in menopausal women [1-3]. A high plasma concentration of low density lipoprotein (LDL) and triglycerides, and a low plasma concentration of high density lipoprotein (HDL) are important predictors in the development of coronary artery disease, contributing to the formation of lesions in the form of plates, which can cause obstruction of the blood flow [4].

Among the mechanisms involved in atherosclerosis are the endothelial changes promoted by dyslipidemia, hypercoagulation, insufficient supply of nitric oxide (NO), oxidative stress, inflammation and endothelial dysfunction [5]. Angiotensin II is recognized as a growth factor that regulates cell proliferation and the fibrosis process. Hence, angiotensin II may initiate inflammation by indirect increase of vascular permeability and recruitment of inflammatory cells [6].

These changes trigger a remodeling in the extracellular matrix (ECM) of the artery. It is characterized by increased thickness of coats due to the gradual destruction of elastic fibers and increased deposit of substances like calcium in the extracellular matrix, promoting increased arterial stiffness, and consequently, changes in its diameter, increase of systolic blood pressure (SBP) and decrease of diastolic blood pressure (DBP) [7-9].

It is known that the practice of physical exercise improves some risk factors, such as the rate of body fat, insulin resistance and high blood pressure, which are associated with increased stiffening of arteries [10]. Moreover, it has been shown that physical exercise has an impact on inflammation, in sympathetic activities and improved endothelial function by increasing blood flow, which leads to increased shear stress, stimulating the release of oxide; it is also known that these factors are involved in arterial stiffening [11]. However, the mechanisms by which exercises affect the properties of the vessels in humans have been poorly studied.

The present study aims to evaluate the effects of moderate aerobic training on biochemical, morphological and physiological parameters in LDL Knockout mice with estrogen deprivation, evaluating the components of the ascending aortic wall.

\section{Materials and Methods}

\section{Animals and Groups}

The study was approved by the Ethics Committee in Research of the Universidade São Judas Tadeu, São Paulo, under protocol number 058/2007. It were used 15 genetically modified female mice, knockout of the low density lipoprotein receptor (LDL-Knockout group) and 15 wild female mice (C57BL/6J) (control group). Both groups were 9 months old, with initial weight ranging from 20 to 30 grams. The mice were kept in cages in a room with controlled temperature $\left(22-24^{\circ} \mathrm{C}\right)$ and a light/dark cycle of $12 / 12$ hours. All mice were fed standard chow and 'ad libitum' water. The animals were randomly divided into six groups (n = 5): sedentary control (SC), sedentary control ovariectomized (SCO), trained control ovariectomized (TCO), LDL-Knockout sedentary (KS), LDL-Knockout sedentary ovariectomized (KOS) and LDL-Knockout trained ovariectomized (KOT). 
Marchon et al.: Moderate Exercise on the Aorta with Experimental Model of LDLKnockout Rats

\section{Ovariectomy}

The ovariectomy was performed at 9 months of age, according to Irigoyen, 2005 [12]. The confirmation of the efficacy of ovariectomy was determined by analysis of vaginal secretions during four consecutive days, with a final analysis at the day of sacrifice of animals.

\section{Physical training}

Physical training began seven days after ovariectomy. The trained groups underwent a protocol of moderate training for 4 weeks on a treadmill with speed and progressive load (one hour per day, $5 \mathrm{x}$ per week and 50-65\% of maximal running speed), according to De Angelis et al., 2004 [13]. The animals were adapted to a treadmill for 10 minutes for three days before the beginning of training. Blood pressure (BP) and body mass were measured weekly. BP was measured in conscious rats by the non-invasive method of tail-cuff plethysmography (Letica LE 5100, Panlab, Spain).

\section{Biochemistry review}

After 5 weeks of the beginning of the protocol, the animals were sacrificed by decapitation. The whole blood was collected in tubes without anticoagulant and centrifuged at $3000 \mathrm{rpm}$ at room temperature for 10 minutes to obtain the serum of each animal. Glucose, total cholesterol and triglycerides were determined using enzymatic colorimetric assay with spectrophotometric reading. The evaluations were performed in duplicate, following the best practices in clinical analysis.

\section{Ascending Aorta}

The ascending aorta was dissected (from heart base to the aorta arch), removed and post-fixed in $4 \%$ paraformaldehyde in $0.1 \mathrm{~mol} / \mathrm{l}$ phosphate buffer, $\mathrm{pH} 7.2$ for $24 \mathrm{~h}$. Aortic rings were dehydrated in graded ethanol concentrations (70,80, 90 and 100\%) and embedded in histological paraffin. The blocks were cut with a microtome ( $5 \mu \mathrm{m}$ - thick section, Leica). Transverse sections were mounted on a glass slide and stained with the Haematoxylin-Eosin, Verhoff-Van Gienson e Picrosirius technique. Four slides with 5 semiserial ( 1 section every $25 \mu \mathrm{m}$ ) sections each, i.e. a total of 20 sections were obtained from each sample. Morphological analysis conducted in transversal aortic sections with a light microscope (Zeiss, x200 and x400 magnifications) permitted the identification of elastic lamellae (Verhoff-Van Gienson stain), smooth muscle constituents (Haematoxylin stain) and collagen fibers (Picrosirius stain).

\section{Morphological/Morphometric Analysis}

Ascendant aorta images were acquired and digitized for off-line morphometric analysis (Image Pro Plus 5.1 software). Four measures per image were obtained at 90,180 and $270^{\circ}$ to estimate intima and media thickness (IMT). The aorta mean cross-sectional area was determined for every animal in each group. The lumen area (a) was estimated by drawing a line over the circle delimited by the intima layer inner interface. The lumen diameter $(d)$ was calculated as: $d=2 \sqrt{a} / \pi$. The mean cross-sectional area of the intima plus media (IMA, intima-media area) was estimated as: IMA $=[\pi(d / 2+$ IMT) $2-\pi(d / 2) 2]$. IMA data were corrected for tissue shrinkage due to fixation and further processing by multiplying by 1.28 (previously determined in a pilot study).

\section{Circumferential Wall Tension and tensile stress}

Circumferential wall tension (CWT) was calculated by Laplace's law as: CWT = MSBP $\times(\mathrm{d} / 2)$, where CWT is expressed in dyne/cm, MSBP is the mean systolic BP (dynes/cm2), and d is the lumen diameter (cm). Mean arterial BP would have been a better value than MSBP, but only the latter was recorded in the present study.

Tensile stress (TS) was computed as: TS = CWT/IMT; where TS is expressed in dyne/cm2, and IMT is the intima plus media thickness in $\mathrm{cm}[14]$.

\section{Stereological Analysis}

Images were captured with a light microscope (Zeiss, $x 400$ magnifications), and transferred to the image analysis program (Axio Vision Software, Zeiss). For volume density of the collagen fibers and elastic lamellae, the photomicrographs of the aorta were analyzed by a stereological test-system with 200 points, and values were expressed as a percentage. The volume density was estimated for the elastic lamellae $(\mathrm{Vv}$ 
Marchon et al.: Moderate Exercise on the Aorta with Experimental Model of LDLKnockout Rats

[lam]) and collagens fibers ( $\mathrm{Vv}[\mathrm{cf}]):(\mathrm{Vv}[$ structure $]=\mathrm{PP}$ [structure]/PT), where PP is the number of points that hit the structure, and PT is the total test-points.

Statistical analysis

The results were presented as mean and standard deviation. Analysis of variance (ANOVA) and Tukey's post-hoc tests were properly applied in data analysis. The significance level for all tests was $p<0.05$.

\section{Results}

Body mass ( $g$ )

There was no significant difference between initial body mass (IM), final body mass (FM) and the difference between the masses (FM-IM). We observed that the menopause promoted increase of visceral adipose tissue (VAT) of 53\% and 16\% in SCO and KOS groups, compared with the SC and KS groups. The exercise caused a reduction of $47 \%$ and $46 \%$ in TCO and KOT groups, when compared to SCO and KOS groups, respectively (Table 1).

\section{Blood Pressure and Heart Rate}

The measurement of the blood pressure signs showed reduced values of diastolic blood pressure (DBP), systolic blood pressure (SBP) and mean arterial pressure (MAP) in the trained groups (TCO and KOT) when compared to the sedentary groups (SCO and KOS). It can also be observed that physical training induced resting bradycardia in the trained groups compared to their respective sedentary groups. Additionally, the KOS group showed an increase in heart rate when compared to the TCO group (Table 2).

Table 1. Initial body mass (IM), final body mass (FM), difference between the masses (FM-IM) and percentage of visceral adipose tissue in relation to final mass (\%VAT). Values represent mean \pm SEM. ${ }^{*} \mathrm{p}<0.05$ vs. Group SC; ${ }^{\#} \mathrm{p}<0.05$ vs. Group SCO; ${ }^{+} \mathrm{p}<0.05$ vs. Group TCO; ${ }^{\mathrm{p}}<0.05$ vs. Group KS; ${ }^{\mathrm{b}} \mathrm{p}<0.05$ vs. Group KOS

\begin{tabular}{lcccccc}
\hline & SC & SC0 & TCO & KS & KOS & KOT \\
\hline IM (g) & $22.65 \pm 0.39$ & $22.39 \pm 0.33$ & $22.42 \pm 0.24$ & $22.89 \pm 0.73$ & $22.47 \pm 0.53$ & $23.90 \pm 0.44$ \\
FM (g) & $24.04 \pm 0.37$ & $23.76 \pm 0.19$ & $23.30 \pm 0.46$ & $23.16 \pm 0.73$ & $24.88 \pm 1.87$ & $25.05 \pm 0.47$ \\
FM-IM(g) & $1.39 \pm 0.20$ & $1.37 \pm 0.4$ & $0.88 \pm 0.52$ & $0.27 \pm 0.13$ & $1.84 \pm 0.71$ & $1.14 \pm 0.23$ \\
VAT\% & $2.36 \pm 0.06$ & $3.62 \pm 0.03^{*}$ & $1.93 \pm 0.06^{*}$ & $2.59 \pm 0.20^{*+}$ & $3.01 \pm 0.03^{* \#+a}$ & $1.62 \pm 0.07^{*}$ *ab \\
\hline
\end{tabular}

Table 2. Diastolic (DBP) and systolic blood pressure (SBP), mean arterial pressure (MAP) and heart rate in the studied groups. Values represent mean \pm SEM at the end of the protocol. ${ }^{*} \mathrm{p}<$ 0.05 The symbols \# to refer the comparisons between the experimental groups of rats. The letter ${ }^{\mathrm{b}}$ was used to show the comparison between the parameters

\begin{tabular}{lcccccc}
\hline & SC & SCO & TCO & KS & KOS & KOT \\
\hline DBP (mmHg) & $85 \pm 2$ & $114 \pm 2.8$ & $89 \pm 3.4^{\# \mathrm{~b}}$ & $102 \pm 2$ & $106 \pm 2.7$ & $88 \pm 2.5^{\# \mathrm{~b}}$ \\
SBP (mmHg) & $110 \pm 2$ & $140 \pm 6$ & $115 \pm 2.1^{\# \mathrm{~b}}$ & $135 \pm 3$ & $143 \pm 3$ & $113 \pm 3.3^{\# \mathrm{~b}}$ \\
MAP (mmHg) & $105 \pm 3$ & $127 \pm 4$ & $106 \pm 2.5^{\# \mathrm{~b}}$ & $116 \pm 3$ & $125 \pm 2.8$ & $101 \pm 2.7^{\# \mathrm{~b}}$ \\
HR (bpm) & $499 \pm 15$ & $596 \pm 16$ & $501 \pm 18^{\#}$ & $589 \pm 16$ & $600 \pm 14+$ & $535 \pm 14^{\mathrm{b}}$ \\
\hline
\end{tabular}

Table 3. Biochemical parameters: glucose $(\mathrm{mg} / \mathrm{dL})$, triglycerides $(\mathrm{mg} / \mathrm{SC}$ $\mathrm{dL})$ and total cholesterol $(\mathrm{mg} / \mathrm{dL})$ in SCO the studied groups. Values represent TCO mean \pm SEM. ${ }^{*}$ p $<0.05$ vs. SC, ${ }^{*}$ p $<0.05$ KS vs. SCO; ${ }^{+} \mathrm{p}<0.05$ vs. TCO; ${ }^{a} \mathrm{p}<0.05$ vs KOS Glucose $(\mathrm{mg} / \mathrm{dL}) \quad \mathrm{Tg}(\mathrm{mg} / \mathrm{dL}) \quad$ Total col. $(\mathrm{mg} / \mathrm{dL})$ KS, ${ }^{b} \mathrm{p}<0.05$ vs. KOS. The symbols \#, $*$ KOT $84.2 \pm 19.8$

$96 \pm 14$

$89.1 \pm 12.9$

$85 \pm 19.2$

$125 \pm 7.4$ $102.9 \pm 44.4$

\begin{tabular}{cc}
$\mathrm{Tg}(\mathrm{mg} / \mathrm{dL})$ & Total col. $(\mathrm{mg} / \mathrm{dL})$ \\
\hline $29.7 \pm 7.6$ & $68.3 \pm 14.7$ \\
$73.2 \pm 16.9^{*}$ & $179.6 \pm 68.9^{*}$ \\
$26.3 \pm 15.5^{\#}$ & $82.7 \pm 16.6^{\#}$ \\
$109.5 \pm 20.7^{* \#+}$ & $220.8 \pm 53.7^{*+}$ \\
$176.5 \pm 7.4^{* \#+\mathrm{a}}$ & $243.4 \pm 5.2^{*+}$ \\
$87 \pm 19.9^{*+\mathrm{b}}$ & $157.4 \pm 32.5^{*+\mathrm{b}}$ \\
\hline
\end{tabular}
and + to refer the comparisons between the experimental groups of rats. The letter $b$ was used to show the comparison between the parameters 
Fig. 1.Transverse sections of KOS mice aorta through atheromatous plaques (p) stained with Verhoeff (A), HE (B) and Picrosirius (C). A, B- The plaque present elastic lamellae (arrows) and nuclei of fibroblasts (arrowheads). C- Collagen fibers of the plaque appear as thick red fibers (arrows) in the tunica media (m) and as thick bundles in the tunica adventitia (a). Bar: $100 \mu \mathrm{m}$.

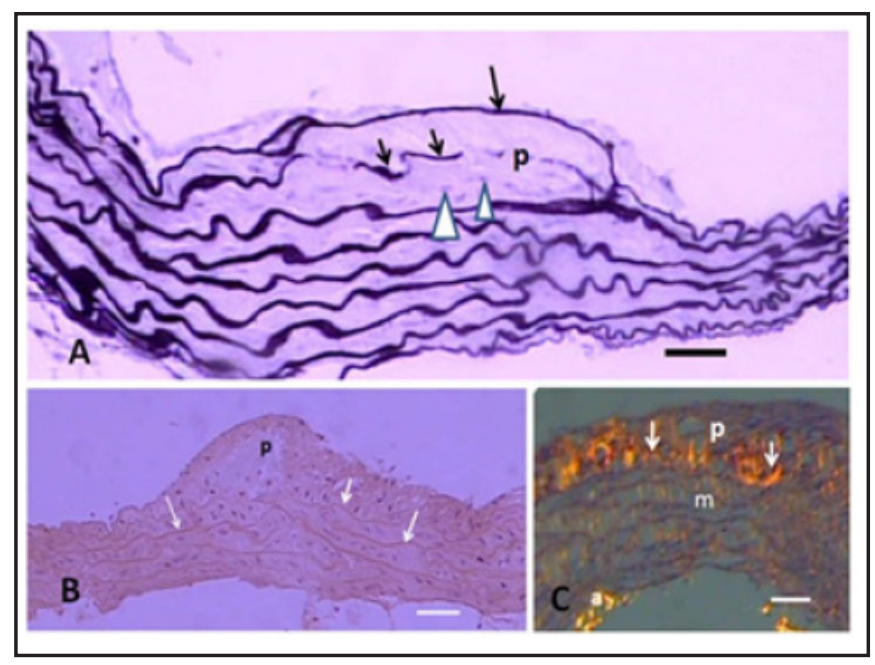

Fig. 2. Photomicrographs of the ascending aorta crosssections with same magnification showing elastic lamellae stained with Verhoeff-Van Gieson. Observe the endothelial cells of KOS group with hypertrophy and a small degree of hyperplasia when compared to the control group. Elastic lamellae in TCO group were more abundant than SC and SCO and less abundant in KOS and KOT than SCO and TCO groups. Bar: $50 \mu \mathrm{m}$.

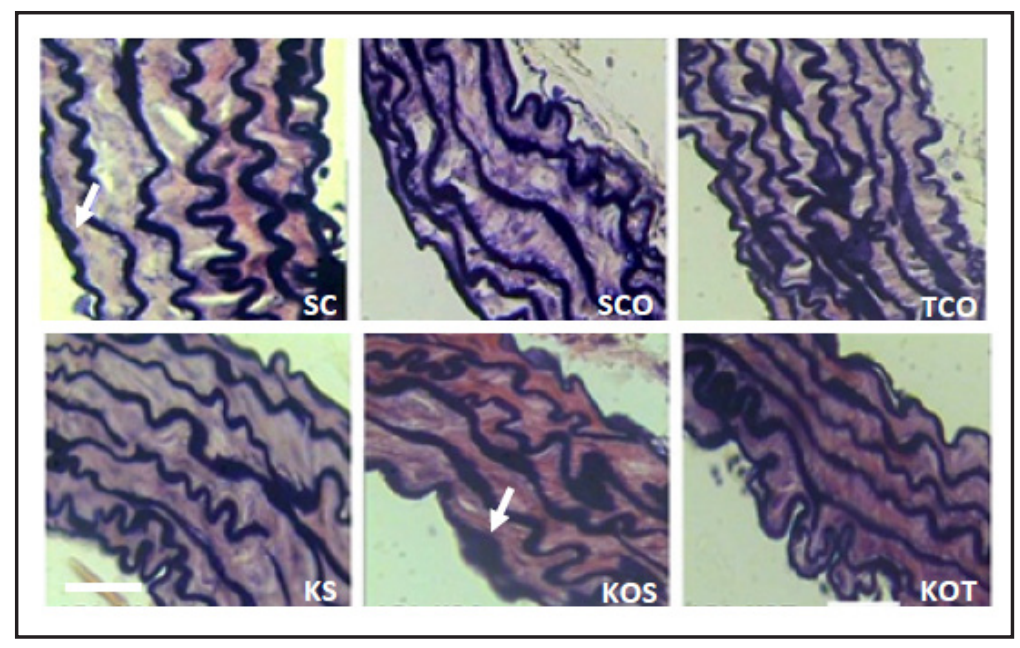

\section{Biochemical parameters}

The parameters of both the TCO group and the KOT group were decreased when compared to the respective control groups. The Table 03 shows all biochemical parameters in each studied experimental group (Table 03).

\section{Morphological data}

The ascending aortas of the KOS group animals showed the presence of typical atheromatous plaques in the intima of the aorta. The plates containing elastic lamellae (Fig. 1A-B), collagen (Fig. 1C) and several nuclei, probably fibroblasts (Fig. 1B). It were found endothelial cells with hypertrophy and small degree of hyperplasia when compared with the control group (Fig 2). No changes were observed in the animals of the control groups.

\section{Morphoquantitative study}

The aortic morphoquantitative analysis are shown in Table 4 and Fig. 2 and 3. Our data show that the ovariectomy has promoted a significant increase $(\mathrm{p}<0.05)$ in volume density of collagen fibers $(\mathrm{Vv}[\mathrm{col}])$ in the SCO group, a trend to increased intima-media thickness (IMT), compared with the SC group. In animals that performed exercises we found significant increase $(\mathrm{p}<0.05)$ in $\mathrm{Vv}[\mathrm{lam}]$; decrease in $\mathrm{Vv}[\mathrm{col}]$ and CWT, and a tendency for decrease both in TS and IMT when compared to the SC groups.

Dyslipidemia induced important changes in the aorta of animals of LDL-K groups, such as significant increase $(\mathrm{p}<0.5)$ in $\mathrm{Vv}[\mathrm{col}]$, IMT and CWT and decrease in $\mathrm{Vv}[\mathrm{lam}]$ when KARGER 


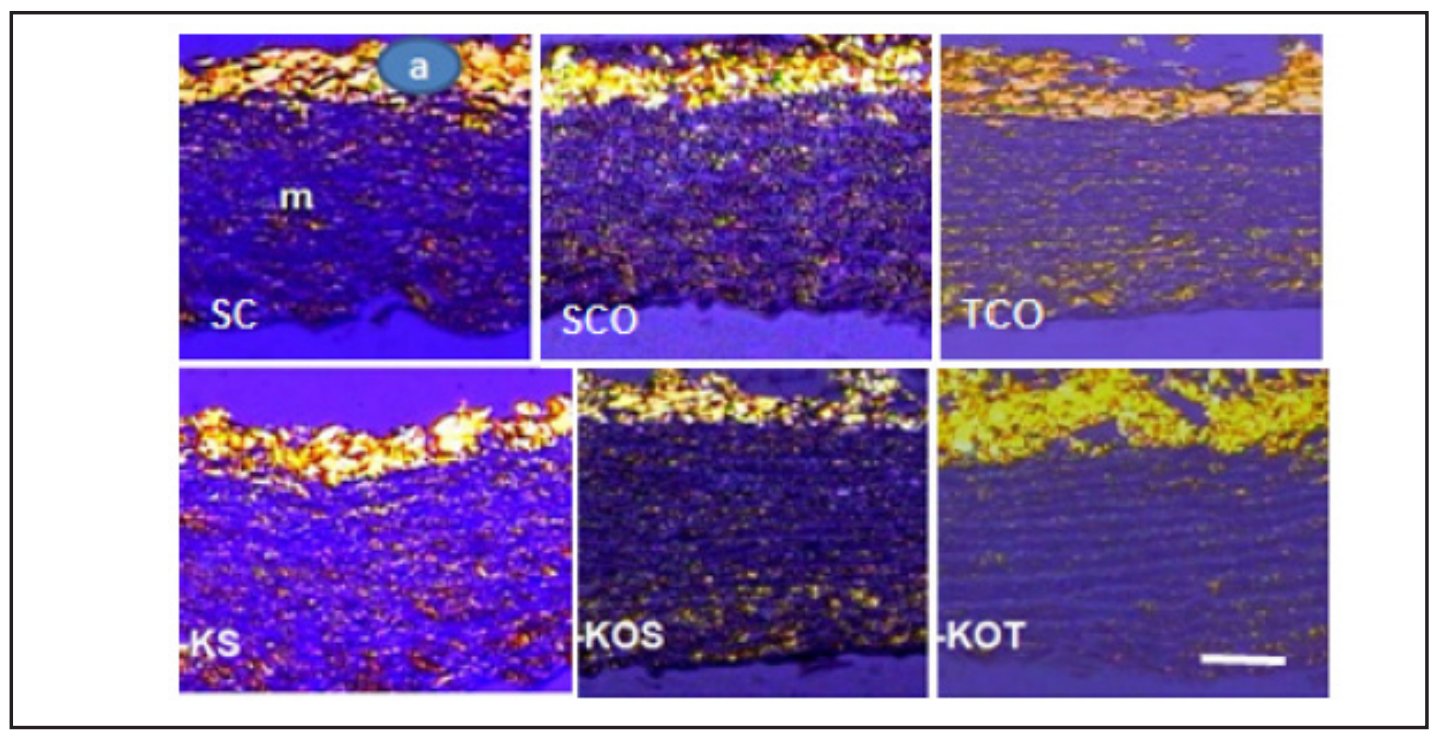

Fig. 3. Transverse sections of mice aorta, stained in Picrosirius and photographed with crossed polarizing filters. Collagen fibers appear as brilliant points in the tunica media (m) and as thick bundles in the tunica adventitia (a). Collagen in the tunica media from SCO and KS groups was more abundant than in the other groups and collagen in KOT is very few. Bar: $100 \mu \mathrm{m}$.

Table 4. Volume density of elastic lamellae Vv[lam], collagen fibers (Vv [cf], intima-media thickness (IMT), area of light ( $\mathrm{CSA}_{\mathrm{L}}$ ), circumferential wall tension (CWT) and tensile stress (TS) of the ascending aorta in the studied groups. Values represent mean \pm SD ${ }^{*} \mathrm{p}<0.05 \mathrm{vs}$. SC, ${ }^{\#} \mathrm{p}<0.05 \mathrm{vs}$. SCO; ${ }^{+} \mathrm{p}<0.05$ vs. TCO; ${ }^{\mathrm{a}} \mathrm{p}<0.05$ vs. KS, ${ }^{\mathrm{b}} \mathrm{p}<0.05$ vs. KOS

\begin{tabular}{|c|c|c|c|c|c|c|}
\hline & $S C$ & SCO & TCO & KS & KOS & KOT \\
\hline$\overline{V v[l a m]}$ & $18.654 \pm 5.321$ & $17.597 \pm 4.111$ & $19.658 \pm 4.377^{\#}$ & $17.920 \pm 4.291$ & $15.856 \pm 3.970^{*} \#$ & $16.539 \pm 5.804^{* *}$ \\
\hline $\mathrm{Vv}[\mathrm{cf}]$ & $2.829 \pm 1.332$ & $3.538 \pm 1.408^{*}$ & $2.826 \pm 1.124^{\#}$ & $3.724 \pm 1.753^{*+}$ & $3.180 \pm 1.550^{\mathrm{a}}$ & $1.038 \pm 0.623^{* *+a b}$ \\
\hline IMT $\left(\mu \mathrm{m}^{2}\right)$ & $137.77 \pm 12.955$ & $162.53 \pm 12.440$ & $147.31 \pm 5.369$ & $146.20 \pm 16.982$ & $160.72 \pm 18.474$ & $188.99 \pm 14.993^{*}+\mathrm{a}$ \\
\hline $\operatorname{CS} A_{L}\left(\mu \mathrm{m}^{2}\right)$ & $6951.1 \pm 642.74$ & $7490.2 \pm 771.33$ & $6864.9 \pm 285.88$ & $6696.3 \pm 969.52$ & $6813.9 \pm 603.62$ & $8692.9 \pm 553.22^{2+a b}$ \\
\hline CWT x $10^{4} \mathrm{dyne} / \mathrm{cm}$ & $12.166 \pm 2.3$ & $16.688 \pm \pm 3.2^{*}$ & $12.56375 \pm 1.1^{*}$ & $14.38425 \pm 4.9$ & $15.5012 \pm 3.2^{*}$ & $15.63355 \pm 2.0^{*}$ \\
\hline TS x $10^{4}$ dyne $/ \mathrm{cm}^{2}$ & $0.0892 \pm 0.014$ & $0.1030 \pm 0.011$ & $0.1039 \pm 0.0001$ & $0.0983 \pm 0.014$ & $0.0978 \pm 0.016$ & $0.0832 \pm 0.0017$ \\
\hline
\end{tabular}

Table 5. Score of expression of angiotensin II and ACE in the studied groups. Values represent mean \pm SEM ${ }^{*} \mathrm{p}<0.05$ vs. SC, ${ }^{*} \mathrm{p}<0.05$ vs. SCO; ${ }^{+} \mathrm{p}<0.05$ vs. TCO; ${ }^{\mathrm{a}} \mathrm{p}<0.05$ vs. KS, ${ }^{\mathrm{b}} \mathrm{p}<0.05$ vs. KOS

\begin{tabular}{ccccccc}
\hline & SC & SC0 & TCO & KS & KOS & KOT \\
\hline ACE & $1.440 \pm 0.219$ & $2.420 \pm 0.232^{*}$ & $2.250 \pm 0.020^{*}$ & $1.740 \pm 0.611$ & $2.826 \pm 0.808^{* a}$ & $3.128 \pm 0.423^{* b}$ \\
AGII & $1.255 \pm 0.433$ & $0.5033 \pm 0.040$ & $2.880 \pm 0.010^{* \#}$ & $2.428 \pm 0.388^{\#}$ & $3.128 \pm 0.463^{* \#}$ & $1.74 \pm 0.523^{\#+b}$ \\
\hline
\end{tabular}

compared to the animals of control groups. We found that exercise caused a decrease of $67 \%$ in $\mathrm{Vv}[\mathrm{col}]$ and $28 \%$ increase in the cross-section area of light $\left(\mathrm{CSA}_{\mathrm{L}}\right)$ and of $18 \%$ in intimamedia thickness (IMT) in animals of the KOT group when compared to KS group.

Expression of angiotensin-converting enzyme (ACE) and angiotensin II

We found that ovariectomy in both control group animals (SCO) and dyslipidemic animals (KOS) produced an increase in the expression of angiotensin-converting enzyme $(\mathrm{p}<0.05)$ of $68 \%$ and $62 \%$ respectively when compared to the respective control groups (SC and KS). We observed that exercising did not change the expression of ACE in animals of control groups (TCO) when compared to the SCO group. In the dyslipidemic group (KOT) 
there was an increase of $11 \%(\mathrm{p}<0.05)$ when compared to the KOS group. Regarding the expression of angiotensin II (AGII) no significant difference was observed between the SCO and SC groups. An increase of $129 \%(\mathrm{p}<0.05)$ was observed in the expression of AGII in the trained control ovariectomized group (TCO) when compared to the SC group.

In dyslipidemic animals, the expression of AGII was increased in both the KS (93\%) as the KOS (521\%) groups, compared with the SCO and SC groups, respectively. The exercise, on its turn, caused a $44 \%$ decrease in the expression of AGII $(\mathrm{p}<0.05)$ compared to KOS group (Table 5).

\section{Discussion}

The relationship between the estrogen and the body mass is widely reported in the literature. When we evaluated the mass in grams of leased animals in the proposed groups according to ovariectomy and moderate aerobic exercise, there was no difference in this parameter in all the animals of the assessed groups. Regarding the percentage of visceral adipose tissue, it was found that ovariectomy leads to an increase in both the LDL-K group and control groups and the exercise reverses this increase. Perhaps, this is because nor obesity, neither overweight have been induced in animals of the studied groups. Still, Zhang et al. 2014 reported the effects on metabolic syndrome in ovariectomized rats with weight gain and also showed the supposed molecular mechanism of action in the treatment with estrogen in adipocytes [15].

Besides the mass in grams, the parameter of blood pressure was checked, and we observed its decrease when the estrogen-suppressed animal was subjected to moderate aerobic exercise. The influence of moderate exercise in relation to blood pressure with low levels of estrogen was also reported by Choi et al., 2013, showing decreased blood pressure in the phase of low estrogen levels in women undergoing moderate physical exercise. The authors reported fluctuation of blood pressure in different phases of the menstrual cycle in order to verify the benefits of moderate exercise at different concentrations of estrogen [16].

Interestingly, when the expressions of angiotensin II and ACE were analyzed in dyslipidemic, ovariectomized animals, we observed an increase in AGII. The AGII is recognized as a growth factor that regulates cell proliferation and fibrosis process. It may initiate inflammation by indirect increase of vascular permeability and recruitment of inflammatory cells [6]. When moderate aerobic exercise is added to these animals, we observed blood pressure decrease with increased expression of ACE and reduction of angiotensin II. Perhaps, physical exercise modulates the expression of these proteins. In the same experimental model, our group showed there is interference of physical exercise on inflammatory pathway by increasing the expression of COX2. The signaling pathway of COX2 can act on angiotensin II and ACE, showing that several proteins can be affected when there are multiple factors associated with menopause, dyslipidemia and physical exercise [5].

The effects of moderate exercise in our experiments also showed benefits when evaluating the concentrations of triglycerides and cholesterol. The parameters of both the TCO group and the KOT group were decreased when compared to the respective control groups. Other authors have reported such effects but not in the group model of LDL- K animals and ovariectomy. The modulation of physical exercise on these parameters is not well defined mainly because the exercise cannot be considered alone per se, but several modulators should be taken into account such as energy expenditure, type of diet (moderate or high fat), time between physical exercise and meals. Such modulators can be evaluated individually or in combination. On the other hand, at least for aerobic exercise, training reduces the concentrations of triglycerides and cholesterol, therefore, exercise sessions should be frequent enough to keep that clinically significant improvement [17].

The effects of ovariectomy were also observed in the morphoquantitative study of the ascending aorta. Our data show that exercise training in the ovariectomized control group (TCO) increased the volume density of elastic lamellae, causing greater aorta compliance 
when compared to animals of the sedentary control group (SC). Corroborating our results, Mereau et al., demonstrated that physical exercise in postmenopausal women improves the compliance of large arteries and reduces fat, making the body composition and LDL cholesterol better, and increasing HDL cholesterol [18]. Perhaps the physical training can increase the bioavailability of NO with the function of preserving the function of the endothelium $[19,20]$.

With respect to collagen fibers, our study showed that the reduction of estrogen levels favors the increase of collagen fibers, which was observed in the SCO group, and that physical exercise was able to keep the results at levels found in the control group (SC). In this context, our findings corroborate Jonason et al. 1998, and Kallikazaros et al. 2002, where these authors relate the decrease in the estrogen hormone with arterial stiffening [21, 22]. Thus, we can hypothesize that physical training contributed to reducing the rigidity of vessels in the estrogen-deprived group that had normal cholesterol levels.

However, when observing the LDL trained ovariectomized group (KOT) there is a significant reduction in the volume density of collagen fibers, which may suggest that when ovariectomy and dyslipidemia associate, the physical training has damaged the extensibility property of the aorta. We could suggest that shear stress stimulated by exercise would be harmful in the presence of a weakened arterial structure due to decrease of the estrogen hormone and of dyslipidemia. In other words, with the mechanical properties of the vessel already damaged, physical training would cause more damages to it by putting pressure on the wall of this vessel. In the other hand, the effects showed in this study to refer the data obtained with training, dyslipidemia and estrogen deprivation. The limitation of these data is the absence of control group without training activity.

Regarding the volume density of elastic lamellae, we found that the trained control ovariectomized group (TCO) presented higher volume density of lamellae in comparison to the SCO and SC groups, suggesting that exercise training was effective in improving the aortic compliance with possible reduction of arterial stiffness, even with ovarian hormone deprivation. However, in the Knockout trained ovariectomized (KOT) and sedentary (KOS) groups, the physical training was not able to reverse the damage caused by the decrease of estrogen and dyslipidemia, corroborating to affect the morphometry of the aorta.

Finally, we conclude that physical training contributed to reducing vessel rigidity and to improvements in vascular compliance, with the increase in volume density of elastic lamellae in the estrogen-deprived groups who had normal cholesterol levels. In relation to estrogen deprivation and dyslipidemia, the same effect was not found probably due to excessive reduction of volume density of collagen fibers. I

Accordingly, further studies are needed regarding the mechanical and functional properties involving dyslipidemia and absence of estrogen for a better understanding of our results.

\section{Disclosure Statement}

The authors declare not conflict of interest.

\section{References}

1 Allen JD, Cobb FR, Kraus WE, Gow AJ: Total nitrogen oxide following exercise testing reflects endethelial function and discriminates health status. Free Radic Biol Med 2006;41:740-747.

2 Leizzi C, Marzullo R, Modena MG: Is menopause a risk factor for ischemic heart disease in women? G Ital Cardiol 2012;12:401-406. 
Marchon et al.: Moderate Exercise on the Aorta with Experimental Model of LDLKnockout Rats

3 Saltiki K, Doukas C, Kanakakis J, Anastasiou E, Mantzou E, Alevizaki M: Severity of cardiovascular disease in women: relation with exposure to endogenous estrogen. Maturitas 2006;12:51-57. doi:10.1016/j. maturitas.2005.12.008.

4 Penalva RA: Perfil lipídico e intensidade de doença aterosclerótica na síndrome coronariana aguda. Arq Brasil Cardiol. 2008;90:24-30.

5 Oliveira F, Maifrino LBM, Jesus GPP, Carvalho JG, Marchon C, Ribeiro AD: The role of cyclooxygenase-2 on endurance exercise training in female LDL-receptor knockout ovariectomized mice. An Acad Bras Cienc 2013;85:155-162.

6 Suzuki Y, Ortega MR, Lorenzo O, Ruperez M, Esteban V, Egido J: Inflammation and angiotensin II. International J Biochem Cell Biology 2003;35: 881-900.

7 Bortolotto LA, Blacher J, Kondo T, Takazawa K, Safar ME: Assessment of vascular aging and atherosclerosis in hypertensive subjects: second derivative of photoplethysmogram versus pulse wave velocity. Am J Hypertens 2000;13:165-171.

8 Kass DA: Ventricular Arterial Stiffening. Integrating the pathophysiology. Hypertension 2005;46:185-93.

9 Girerd X, Mourad J J, Boutouvrie P, Benetos A, Laurent S, Safar M: Effects of aging on arterial function in man. Minerva Cardioangio 2007;5:497-502.

10 Stehouwer C D, Ferreira I: Diabetes, lipids and other cardiovascular risk factors. In: Safar M E, O'Rourke M F (eds): Arterial stiffness in hypertension - handbook of hypertension. Amsterdam: Elsevier, 2006, vol. 23, pp 427-456

11 Tanaka H, Safar M E: Influence of lifestyle modification on arterial stiffness and wave reflections. Am J Hypertension 2005;18:137-144.

12 Irigoyen MC, Paulini J, Flores LJF, Flues K, Bertagnolli M, Moreira ED, Consolim-Colombo F, Belló-Klein A, De Angelis K: Exercise Training Improves Baroreflex Sensitivity Associated With Oxidative Stress Reduction in Ovariectomized Rats. J Hypertension 2005;46:998-1003.

13 De Angelis K, Santos MSB, Irigoyen MC: Sistema nervoso autônomo e doença cardiovascular. Rev da Soc de Cardiol do Rio Grande do Sul 2004;13:1-7.

14 Mendonca LS, Fernandes-Santos C, Mandarim-de-Lacerda CA: Cardiac and aortic structural alterations due to surgicallyinduced menopause associated with renovascular hypertension in rats. Int J Exp Pathol 2007;88:301-309.

15 Zhang R, Su D, Zhu W, Huang Q, Liu M, Zhang Y, Xue Y, Li D, Zhao A, Liu Y: Estrogen suppresses adipogenesis by inhibiting S100A16 expression. J Mol Endocrinol 2014;52:235-244.

16 Choi HM, Stebbins CL, Nho H, Kim MS, Chang MJ, Kim JK: Effects of Ovarian Cycle on Hemodynamic Responses during Dynamic Exercise in Sedentary Women. Korean J Physiol Pharmacol 2013;17:499-503.

17 Maraki MI, Sidossis LS: The latest on the effect of prior exercise on postprandial lipaemia. Sports Med 2013;43:463-481.

18 Moreau KL, Gavin KM, Plum AE, Seals DR. Oxidative stress explains differences in large elastic artery compliance between sedentary and habitually exercising postmenopausal women. North Am Menopause Soc 2006;13:951-958.

19 Lima SM, Aldrigui JM, Consolim-Colombo FM, Mansur P, Rubira MC, Krieger EM, Ramires JA: Administration of 17 beta-estradiol improves endothelium-dependent vasodilation in postmenopausa. Maturitas 2005;50:266-274.

20 Lee J, Cho HS, Kim DY, Cho JY, Chung JS, Lee HK, Seong NH, Kim WK: Combined effects of exercise and soy isoflavone diet on paraoxonase, nitric oxide and aortic apoptosis in ovariectomized rats. Appetite 2012;58:462-469.

21 Jonason T, Henriksen E, Kangro T, Vessby B, Ringqvist I: Menopause is associated with the stiffness of the common carotid artery in 0 year old women. Clin Physiol 1998;18:149-155.

22 Kallikazaros I, Tsioufis C, Zambaras P, Stefanadis C, Toutouzas P: Conjugated estrogen administration improves common carotid artery elastic proporties in normotensive postmenopausal women. Clin Cardiol 2002;25:167-172. 